\title{
Single Cell Level Dielectrophoretic Responses \& Dielectrophoretic Deformations of Monocytes to Quantify Population Heterogeneity
}

\author{
Esra Sengul $^{\dagger}$, Osman Kara, Yagmur Yildizhan, Rodrigo Martinez-Duarte ${ }^{\dagger}$, and Meltem Elitas ${ }^{\dagger}$, \\ ${ }^{\dagger}$ Member, IEEE
}

\begin{abstract}
Single-cell dielectrophoretic movement and dielectrophoretic deformation of monocyte cells were interrogated applying $20 \mathrm{~V}_{\mathrm{pp}}, 50 \mathrm{kHz}$ to $1 \mathrm{MHz}$ signal in the 3D carbon electrode array. Heterogeneity of the monocyte population is shown in terms of the crossover frequencies, translational movement, and deformation index of the cells. The results presented that crossover range for monocytes was 100 $\mathrm{kHz}-200 \mathrm{kHz}$, the translational movement of the cells was rapidly altered when the initial positions of the cells were in the negative dielectrophoretic region. Finally, the deformation index of the monocyte population varied from 0.5 to 1.5 .
\end{abstract}

\section{INTRODUCTION}

Monocytes compose 5\% of leukocytes in the human body and hold an important role in the immune system. Understanding the origin and fate of monocytes is crucial to underline their behavior in the cancer microenvironment. Monocytes have different roles in tumor progression from tumor formation to invasion, metastasis, and angiogenesis [1, 2]. In particular, tumor-associated macrophages (TAM) are one of the key players in tumor development and progression in many types of cancer $[3,4,5]$. TAM are monocyte-derived immune cells and are classified based on their cytokines and immune functions as M1- and M2-polarized subtypes [6]. The M1 subtype suppresses while the M2 subtype promotes tumor growth. Therefore, understanding population heterogeneity, isolation and characterization of monocytes and monocyte subpopulations are required for precise diagnostics of tumor grades and development of personalized medicine.

Here, we use Dielectrophoresis (DEP) to characterize such population heterogeneity. Since DEP does not require any prelabelling of the cells, it allows for direct characterization of monocyte subtypes based on their intrinsic properties without altering their genotype and phenotype. In addition to DEP [7], there are several well-established cell separation and characterization methods. Fluorescence-activated cell sorting (FACS) $[8,9]$ and magnetic-activated cell sorting (MACS)

M. E. Author is with the Sabanci University, Istanbul, 34956 Turkey (corresponding author to provide phone: +90 0216483 9568; e-mail: melitas@sabanciuniv.edu).

E. S. Author was with Sabanci University, Istanbul, 34956 Turkey (email: sengulesra@sabanciuniv.edu).

O. K. Author was with, Zonguldak Bulent Ecevit University Faculty of Sciences, 67100 Zonguldak, Turkey (e-mail: karaosmannn1 @gmail.com).
[10] techniques are among the widely used cell enrichment and isolation methods. Moreover, hydrodynamic forces [11, 12], optical tweezers [13] and free flow acoustophoresis (FFA) [14] have been used for cell enrichment and manipulation.

DEP has many advantages, such as cells remain viable, maintain their genetic and phenotypic properties [15], and it is a low-cost technique in terms of not requiring fluorescent labels on the cells $[16,17,18]$. Dielectrophoretic responses of cells can be monitored when the cells are exposed to the nonuniformly distributed electric field in the low-conductive suspension medium. Hence, cells are experienced either attractive dielectrophoretic forces, or repulsive dielectrophoretic forces according to their polarizability [7, 19]. DEP has been used not only for cell isolation but also for manipulation of cells [20,21, 22], DNA [23] and proteins [24].

Dielectrophoretic forces have been generated by patterned electrodes in microfluidic channels. In the earlier studies, various geometries and dimensions have been tested for electrodes in order to optimize dielectrophoretic stimuli of particles or biologic samples [25]. Recent studies in dielectrophoresis community mostly focused on rapid detection, low cost and high-throughput cell enrichment [16, $26,27,28]$. Hence, the manufacturing of dielectrophoresis platforms, low-cost material usage, disposable and environment-friendly products become important.

Furthermore, we used 3-dimensional carbon electrodes (3D carbon DEP) to create dielectrophoretic forces that might reflect the heterogeneity of monocyte populations [29, 30]. We quantified the displacement and deformation of monocytes under the influences of dielectrophoretic forces when $20 \mathrm{~V}_{\text {peak- }}$ to-peak $\left(\mathrm{V}_{\mathrm{pp}}\right)$ voltage with frequencies ranging from $50 \mathrm{kHz}$ to 1 MHz have been applied. We analyzed the position of the single cells in the 3D carbon DEP chip to determine the dielectrophoretic responses of the single monocyte cells. We measured the height and width of the cells to calculate the deformation index of the cells [31].

Y.Y. was with Sabanci University, Istanbul, 34956, Turkey. She is now with the Division of Mechatronics, Biostatistics and Sensors, KU Leuven 3001 Belgium (e-mail:yagmur.yildizhan@kuleuven.be).

R. M-D. Author is with the Mechanical Engineering Department, Clemson University, Clemson, SC 29634 USA, (e-mail: rodrifm@clemson.edu). 


\section{MATERIALS AND METHODS}

\section{3D CARBON-DEP DEVICE MANUFACTURING}

The fabrication of 3D carbon-microelectrodes has been described in detail [32, 33]. In short, a two-step photolithography process with SU-8 (Gersteltec, Pully, Switzerland) was implemented on a silicon wafer to create the $3 \mathrm{D}$ precursor structure to the micro-electrodes. The photoresist pattern was then carbonized at $1000^{\circ} \mathrm{C}$ in a nitrogen atmosphere. The carbon electrode array featured 218 intercalated rows with 14 or 15 electrodes each. Individual electrodes had a height of $100 \mu \mathrm{m}$ and a diameter of $50 \mu \mathrm{m}$. A thin layer of SU-8 was then applied to isolate the planar connectors and make the bottom channel plane. From a 127 mm thick double-sided pressure-sensitive adhesive (PSA, Switchmark 212R, Flexcon, Spencer, MA, USA), a $1.8 \mathrm{~mm}$ wide, $3.2 \mathrm{~cm}$ long channel was cut and glued to a pre-drilled polycarbonate [34]. This arrangement was then manually placed around the carbon-electrode array and closed using a rolling press.

\section{DEP Buffer Preparation}

A DEP buffer with low electrical conductivity was prepared according to previous formula [35], 8.6\% sucrose (Product no: LC-4469.1, neoFroxx, Hesse, Germany), 0.3\% glucose (CAS Number 59-99-7, Sigma-Aldrich, Darmstadt, in accordance with the pre-specified formula [34], Germany) and $0.1 \%$ dilution. Bovine Serum Albumin in distilled water (BSA, Product Code: P06-1391050, PAN-Biotech, Aidenbach, Germany). The conductivity of the final suspension was $20 \mu \mathrm{S}$ / $\mathrm{cm}$ as measured (Corning Model 311 Portable Conductivity Meter, Cambridge Scientific Products, Watertown, MA, USA).

\section{Cell Preparation}

U937 monocyte cells are human myeloid leukemia cell lines obtained from the American Type Culture Collection (ATCC, Manassas, VA, USA) were maintained in $10 \%$ fetal bovine serum (FBS; PAN-Biotech, Aidenbach, Germany) RPMI (Roswell Park Memorial Institute) - 1640 medium (Product Number: P04-18047, PAN-Biotech, Aidenbach, Germany) $37^{\circ} \mathrm{C}$ from (EC $160 \mathrm{CO} 2$ Incubator, Nuva, Ankara, Turkey) in a humidified incubator at $5 \% \mathrm{CO}_{2}-95 \%$ air atmosphere in plastic bottles.

Cells were centrifuged at $3000 \mathrm{rpm}$ (Z601039 - Hettich ${ }^{\circledR}$ EBA 20 centrifuge, Merck, Darmstadt, Germany) for 5 minutes to remove the remaining culture medium and resuspended twice in DEP buffer.

\section{Experimental Setup}

The experimental setup consisted of the signal generator (Model: GFG-8216A, GW Instek, New Taipei City, Taiwan), an oscilloscope (Part Number: 54622D, Agilent Technologies, Santa Clara, CA, USA) to create the electric field. A desktopacquired, upright microscope (Model: Nikon ME600 Eclipse, Nikon Instruments Inc., Melville, NY, USA) to acquire and analyze cells for monitoring, recording and analyzing images (Hewlett-Packard Company, Palo Alto, CA, USA), a programmable syringe pump to flow cells and DEP buffer (Model: NE-1000, New Era Pump Systems Inc, Farmingdale, NY, USA) and our 3D carbon-DEP device. To create a reservoir, 20-200 $\mu \mathrm{L}$ pipette tips (Manufacturer ID: 3120000917, Eppendorf, Hamburg, Germany) used as a reservoir at the inlet and outlet of the microchannel. Tygon micro-perforated tube (Manufacturer ID: AAQ02103-CP S54-HL, Cole-Parmer, Vernon Hills, IL, USA) was used to connect the syringes and microchannels of the $3 \mathrm{D}$ carbon-DEP chip.

\section{Experimental Procedure}

First, the 3D carbonDEP chip was sterilized with $70 \%$ Ethanol and rinsed with DI (deionized) water. Next, the chip was filled with DEP buffer and all the bubbles on the chip were removed. $40 \mu \mathrm{L}$ of the cell suspension was loaded into the chip using a syringe pump. The flow rate was $10 \mu \mathrm{L} / \mathrm{min}$. When the cells reached the electrode area, the flow was stopped, and the cells were released for 30 seconds. A signal with $20 \mathrm{Vpp}$ frequencies ranging from $50 \mathrm{kHz}-1 \mathrm{MHz}$ was then applied using the function generator [36]. The movement of the cells was generated due to the dielectrophoretic forces, there was no fluid flow in the microfluidic device.

\section{Image Acquisition and Analysis}

All images were recorded using a $10 \times$ lens mounted on the Nikon Eclipse vertical optical microscope. Then, the acquired images were analyzed using ImageJ (version 2.0 National Institutes of Health, Rockville, MD, USA). Since each image considers the location information of the cells for a given frequency, we measured the positions of the cells in the electrode array. Afterwards, we evaluated the location of the cells according to type and magnitude of the dielectrophoretic forces they experienced. The dielectric forces are valued from 3 to -3 . The dielectric force on 3 considered as the strongest pDEP and -3 as the strongest nDEP. We used VideoLAN Client (VLC, VideoLAN version 1.8, Paris, France) to convert image sequences into the movies.

\section{RESULTS}

DEP allows characterization of cells based on their electrical polarizability and the resulting specific translational dielectrophoretic forces $\left(\mathrm{F}_{\mathrm{DEP}}\right)$ given in (1).

$$
\mathrm{F}_{\mathrm{DEP}}=2 \pi \varepsilon_{\mathrm{m}} \mathrm{r}^{3} \operatorname{Re}\left[\mathrm{f}_{\mathrm{CM}}\right] \nabla \mathrm{E}_{\mathrm{rms}}^{2}
$$

where applied electric field $(\mathrm{E})$ and the permittivity of the suspending medium $\left(\varepsilon_{\mathrm{m}}\right)$ are the parameters that equally affects all types of cells in the electrode array. However, the real part of the Clausius-Mossotti factor $\operatorname{Re}\left[\mathrm{f}_{\mathrm{cm}}(\omega)\right]$ introduces specificity based on the membrane thickness (d), radius $(r)$, conductivity $\left(\sigma_{\text {int }}\right)$ and permittivity of the cells $\left(\varepsilon_{\text {int }}\right)$ and their surrounding buffer, in $(\mathbf{2}, \mathbf{3})$,

$$
\mathrm{f}_{\mathrm{cm}}=\frac{\varepsilon_{\mathrm{eff}}^{*}-\varepsilon_{\mathrm{m}}^{*}}{\varepsilon_{\mathrm{eff}}^{*}+2 \varepsilon_{\mathrm{m}}^{*}}
$$

where $\varepsilon_{\mathrm{m}}^{*}$ is the complex permittivity of the medium, $\varepsilon_{\mathrm{eff}}^{*}$ is the effective permittivity of the cell using (3) and (4), according to single-shell model, the imaginary number (j) [25, 26].

$$
\varepsilon^{*}=\varepsilon-\frac{j \sigma}{\omega}
$$




$$
\varepsilon_{\mathrm{eff}}^{*}=\varepsilon_{\mathrm{mem}}^{*} \frac{\left(\frac{\mathrm{r}}{\mathrm{r}-\mathrm{d}}\right)^{3}+2 \frac{\varepsilon_{\mathrm{int}}^{*}-\varepsilon_{\mathrm{mem}}^{*}}{\varepsilon_{\mathrm{int}}^{*}+2 \varepsilon_{\mathrm{mem}}^{*}}}{\left(\frac{\mathrm{r}}{\mathrm{r}-\mathrm{d}}\right)^{3}-\frac{\varepsilon_{\mathrm{int}}^{*}-\varepsilon_{\mathrm{mem}}^{*}}{\varepsilon_{\mathrm{int}}^{*}+2 \varepsilon_{\mathrm{mem}}^{*}}}
$$

These intrinsic dielectric properties determine the polarizability of the cells under the applied non-uniform electric field. Hence, each specific cell type will experience a specific DEP force. When the $\operatorname{Re}\left[\mathrm{f}_{\mathrm{cm}}(\omega)\right]$ is positive, the cells migrate towards regions of high electric field gradient in a phenomenon known as positive-DEP (pDEP). When the $\operatorname{Re}\left[\mathrm{f}_{\mathrm{cm}}(\omega)\right]$ is negative, negative DEP (nDEP) occurs, and cells migrate away from the field gradient. The frequency when the cells experience zero $\mathrm{F}_{\mathrm{DEP}}$ is defined as the crossover frequency $(\mathrm{CF})$.

In order to determine the dielectrophoretic responses of the cells, the location of each cell was measured in each frequency when the electric field was applied. Fig. 1 illustrated the number of cells those experienced strong pDEP (3), pDEP (2), weak pDEP (1), CF (0), weak nDEP (-1), nDEP (-2), strong nDEP (-3) at $50 \mathrm{kHz}, 100 \mathrm{kHz}, 200 \mathrm{kHz}, 300 \mathrm{kHz}$, $400 \mathrm{kHz}$ and $1000 \mathrm{kHz}$ frequencies when $20 \mathrm{~V}_{\mathrm{pp}}$ was applied.

In order to quantify heterogeneity of the monocyte population in terms of their dielectrophoretic behaviors at least 50 single cells were analyzed for each case. Fig. 2 shows the trajectory of single monocytes when they started their motion at CF, weak nDEP (-1), nDEP (-2) and strong $\mathrm{nDEP}$ $(-3)$. When the cells started their movement at pDEP, they remained at $\mathrm{pDEP}$ region without moving to $\mathrm{CF}$ or $\mathrm{nDEP}$.

We followed 80 monocyte cells in the electrode array when DEP forces were acting on them. There was no fluid flow in the electrode array. We categorized cells according to their initial DEP responses. Fig. 2a presents the DEP behavior of the cells when they started their motion at CF.

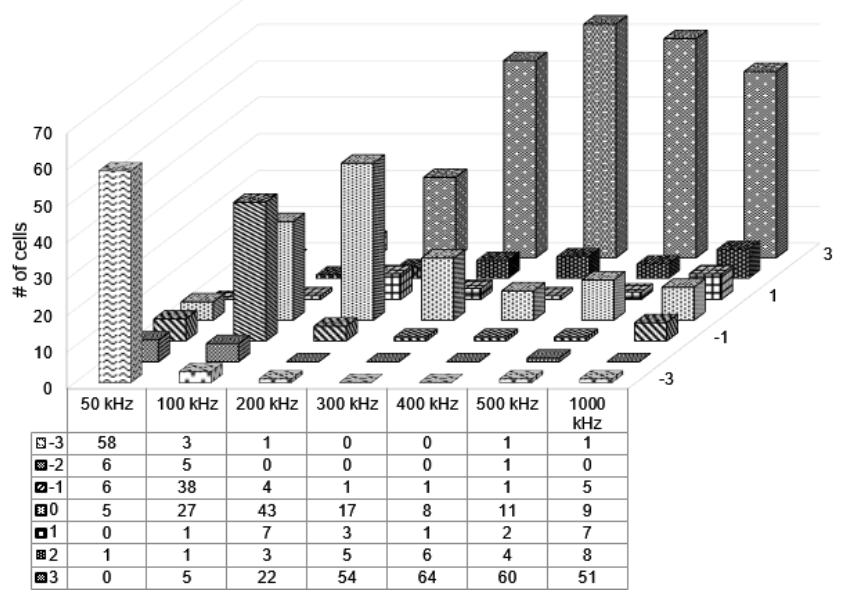

Figure 1. DEP response of the monocyte population. The number of monocyte cells was counted at strong pDEP (3), pDEP (2), weak pDEP (1), $\mathrm{CF}$, weak $\mathrm{nDEP}(1)$, $\mathrm{nDEP}(2)$, strong $\mathrm{nDEP}(-3)$ regions of the electrode array when $50 \mathrm{kHz}$ to $1 \mathrm{MHz}$ frequency, $20 \mathrm{~V}_{\mathrm{pp}}$ was applied. 80 cells for each frequency was counted.
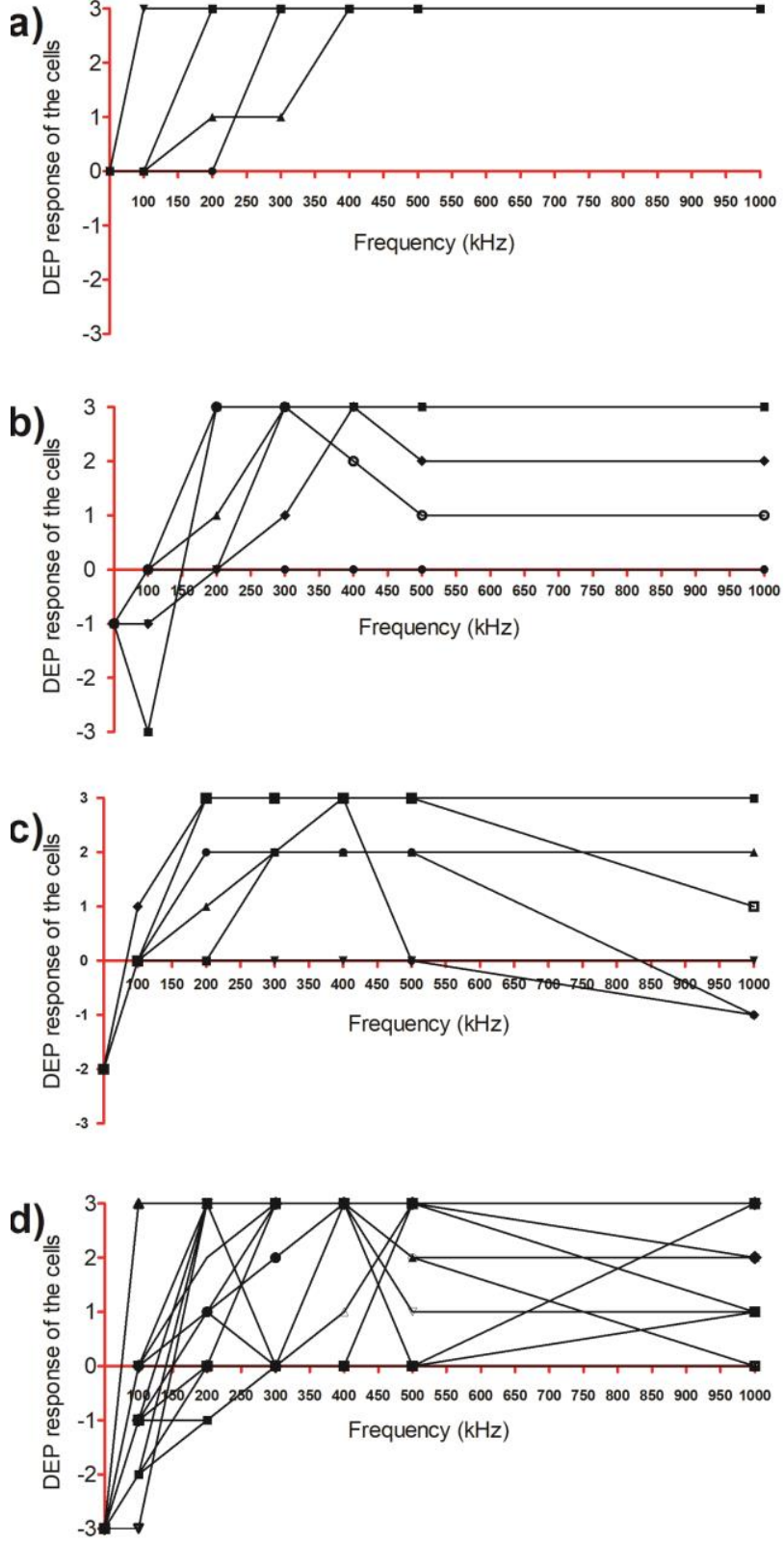

Figure 2. The trajectory of single monocytes in the presence of the nonuniform electric field with $20 \mathrm{~V}_{\mathrm{pp}}, 100 \mathrm{kHz}$ to $1 \mathrm{MHz}$ frequency range. DEP responses of the cells when they started their behavior in the a) $\mathrm{CF}, \mathrm{b}$ ) weak $\mathrm{nDEP}, \mathrm{c}$ ) $\mathrm{nDEP}, \mathrm{d}$ ) strong $\mathrm{nDEP}$ regions of the electrode array.

Next, we evaluated the responses of the monocytes when they started at weak nDEP (Fig. 2b), nDEP (Fig. 2c), and strong nDEP (Fig. 2d). As mentioned above, we used fluid flow to load the cells into the microfluidic device, there was no any fluid flow in the electrode array during the experiments.

In the absence of the fluid flow, in the presence of the DEP forces, the diameters of the cells changed as shown in Fig. 3. We measured the height $(\mathrm{H})$ and width $(\mathrm{W})$ of the 50 monocyte cells for each frequency ranging from $100 \mathrm{kHz}$ to 1 $\mathrm{MHz}$ when $20 \mathrm{~V}_{\mathrm{pp}}$ was applied. 


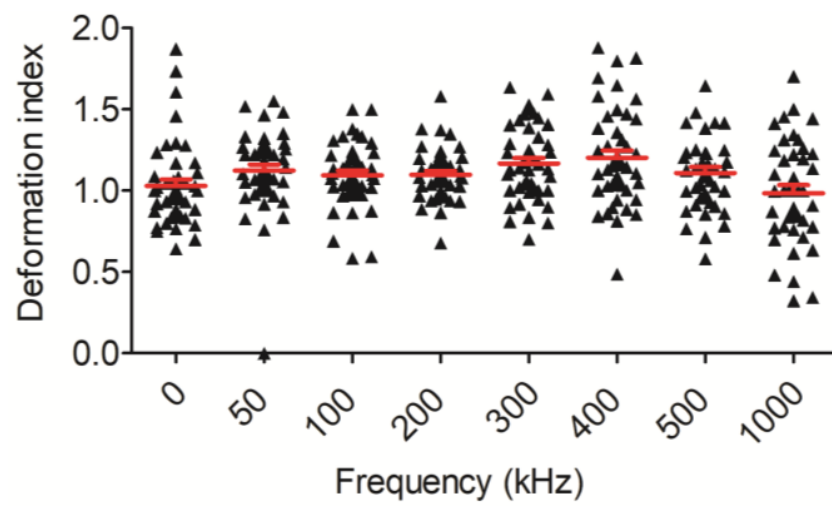

Figure 3. DEP deformation indexes for monocyte population. Deformation index was calculated by measuring the height and width of the single monocytes. 50 monocytes were analyzed for each frequency (Supplementary movie). Red lines show means and standard error values.

We calculated the deformation index (DI) of each monocyte using the following formula (5) as described in [26].

$$
D I=\frac{H}{W}
$$

Fig. 4, as a representative, shows the deformation of single monocytes in the presences of DEP forces. We measured the height and width of three monocytes frame-byframe to follow the change of their deformation index for the varying DEP forces. We labelled monocytes in red, blue and yellow lines in the images, Fig. 4a. Fig. 4b displays the change of monocyte deformation index (labelled in Fig. 4a) according to applied dielectrophoretic forces.

a)
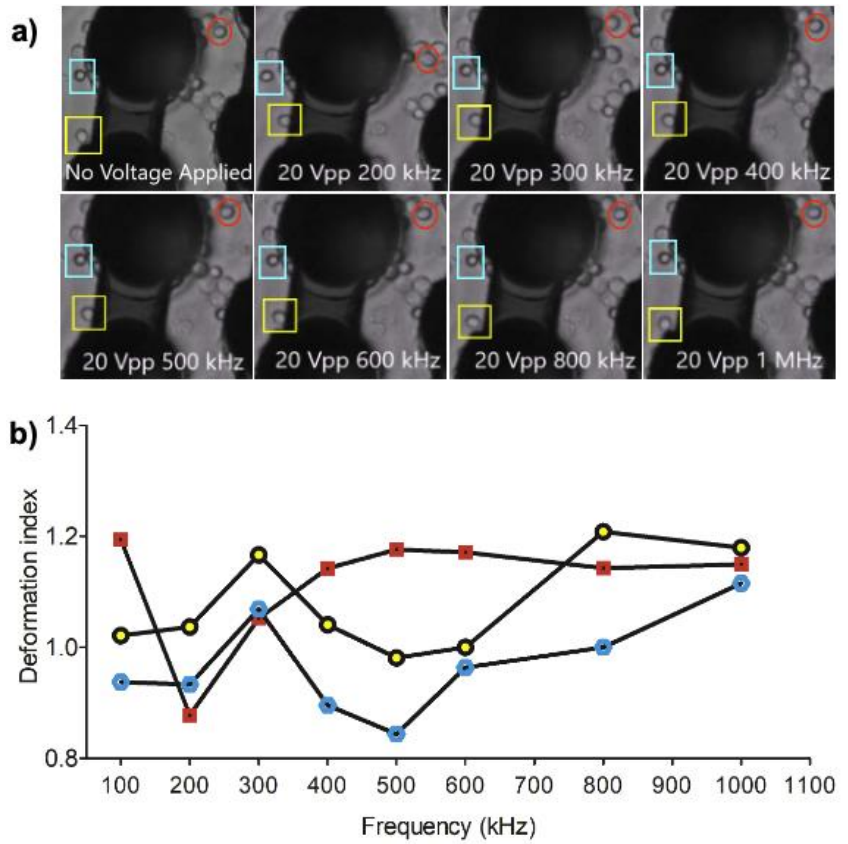

Figure 4. DEP deformation trend for the single monocytes. a) Snapshots of electrode arrays with labeled cells that shows motion and deformation of cells during the applied electric field (Supplementary movie). b) The change in the deformation index for the labeled cells in the electrode array.

\section{CONCLUSION}

Here we presented experiments that quantify heterogeneity of monocytes according to their intrinsic dielectrophoretic properties in terms of dielectrophoretic movement and dielectrophoretic deformation index at the single-cell level. We conclude that the change of the deformation index is correlated with the generated DEP forces on the monocytes. When the cells are attracted by strong pDEP, their height decreases, and width increases then their deformation index become less than 1 . When the cells are at the crossover frequency, the existing DEP forces are so weak, almost zero, therefore, deformation index of the cells was closer to one. When the electrodes repel the cells, their deformation index increases. In this study, we particularly emphasize on DEP generated deformation index of monocytes since monocytes and other white blood cells are capable of infiltrating different types of tissue. The intrinsic cellular heterogeneity of white blood cells might phenotypically affect their plasticity that we can quantity using DEP.

\section{REFERENCES}

1. M. J. Pittet, \& F. K. Swirski, "Monocytes link atherosclerosis and cancer", European Journal of Immunology, 41(9), 2519-2522, 2011.

2. A. Etzerodt, K. Tsalkitzi, M. Manieck, W. Damsky, M. Delfini, E. Baudoin ... T. Lawrence, "Specific targeting of CD163 TAMs mobilizes inflammatory monocytes and promotes $\mathrm{T}$ cell-mediated tumor regression" The Journal of Experimental Medicine, 216(10), 2394-2411, 2019

3. C. Atri, F. Guerfali, \& D. Laouini, "Role of Human Macrophage Polarization in Inflammation during Infectious Diseases" International Journal of Molecular Sciences, 19(6), 1801, 2018.

4. A. Yuan, Y.J. Hsiao, H-Y Chen, H.W. Chen, C-C. Ho, Y-Y. Chen, et al., "Opposite Effects of M1 and M2 Macrophage Subtypes on Lung Cancer Progression”. Sci Rep., 5:14273, 2015

5. W. Ying, P. S. Cheruku, F. W. Bazer, S. H. Safe, \& B. Zhou, "Investigation of Macrophage Polarization Using Bone MarrowDerived Macrophages", J. Vis. Exp. (76), e50323, 2013.

6. M. Zhang, G. Hutter, S. A. Kahn, T. D. Azad, S. Gholamin, C. Y $\mathrm{Xu}, \ldots$ S. H. Cheshier, "Anti-CD47 Treatment Stimulates Phagocytosis of Glioblastoma by M1 and M2 Polarized Macrophages and Promotes M1 Polarized Macrophages In Vivo", Plos One, 11(4), 2016.

7. A. Jaffe, \& J. Voldman, "Multi-frequency dielectrophoretic characterization of single cells", Microsystems \& Nanoengineering, 4(1), 2018.

8. M. J. Fulwyler, "Particle Separator", US 80584, issued 1965-06-01

9. M. J. Fulwyler, "Electronic Separation of Biological Cells by Volume", Science, 150(3698), 910-911, 1965.

10. S. Miltenyi, W. Müller, W. Weichel, \& A. Radbruch, "High gradient magnetic cell separation with MACS", Cytometry, 11(2), 231-238, 1990

11. J. Alvankarian, A. Bahadorimehr, B. Yeop Majlis, "A pillar-based microfilter for isolation of white blood cells on elastomeric substrate", Biomicrofluidics, vol. 7, no. 1, pp. 014102, 2013.

12. R.-G. Wu, H.-C. Tseng, H.-Y. Chang, \& F.-G. Tseng, "Label-free blood cells separation and enrichment from whole blood by highthroughput hydrodynamic and inertial force", 2013 Transducers \& Eurosensors XXVII: The 17th International Conference on Solid-State Sensors, Actuators and Microsystems (Transducers \& Eurosensors XXVII), 2013.

13. H. Zhang, \& K.-K. Liu, "Optical tweezers for single cells. Journal of The Royal Society Interface”, 5(24), 671-690, 2008.

14. F. Petersson, L. Åberg, A.-M. Swärd-Nilsson,\& T. Laurell, "Free Flow Acoustophoresis: Microfluidic-Based Mode of Particle and Cell Separation", Analytical Chemistry, 79(14), 5117-5123, 2007. 
15. H. A. Pohl, K. Pollock, \& J. S. Crane, "Dielectrophoretic force: A comparison of theory and experiment", Journal of Biological Physics, $6(3-4), 133-160,1978$.

16. S. A. Faraghat, K. F. Hoettges, M. K. Steinbach, D. R. V. D. Veen, W. J. Brackenbury, E. A. Henslee, M. P. Hughes. "High-throughput, low-loss, low-cost, and label-free cell separation using electrophysiology-activated cell enrichment." Proceedings of the National Academy of Sciences, 114(18), 4591-4596, 2017.

17. Elitas, M.; Martinez-Duarte, R.; Dhar, N.; McKinney, J.D.; Renaud, P. Dielectrophoresis-based purification of antibiotic-treated bacterial subpopulations. Lab Chip 2014, 14, 1850-1857.

18. Yang, J.; Huang, Y.; Wang, X.; Wang, X.B.; Becker, F.F.; Gascoyne, P.R.C. Dielectric properties of human leukocyte subpopulations determined by electrorotation as a cell separation criterion. Biophys. J. 1999,76, 3307-3314.

19. J. Nieuwenhuis, A. Jachimowicz, P. Svasek, \& M. Vellekoop, "Optimization of microfluidic particle sorters based on dielectrophoresis", IEEE Sensors Journal, 5(5), 810-816, 2005.

20. X.B. Wang, Y. Huang, X. Wang, F. F. Becker, P. R.Gascoyne, "Dielectrophoretic manipulation of cells with spiral electrodes", Biophys J., 72(4):1887-1899, 1997

21. M. R. Buyong, A. A. Kayani, A. A. Hamzah, B. Yeop Majlis, "Dielectrophoresis Manipulation: Versatile Lateral and Vertical Mechanisms", Biosensors (Basel); 9(1):30, 2019

22. N. Lewpiriyawong, C. Yang, \& Y. C. Lam. "Continuous sorting and separation of microparticles by size using $\mathrm{AC}$ dielectrophoresis in a PDMS microfluidic device with 3-D conducting PDMS composite electrodes." Electrophoresis, 31(15), 2622-2631, 2010.

23. J, Regtmeier, TT, Duong, R. Eichhorn, D. Anselmetti, A. Ros. "Dielectrophoretic manipulation of DNA: Separation and polarizability." Anal Chem.; 79(10):3925-32., 2007

24. A. Nakano, A. Ros." Protein dielectrophoresis: advances, challenges, and applications." Electrophoresis. 34(7):1085-1096, 2013.

25. H. Zhang, H. Chang, \& P. Neuzil. "DEP-on-a-Chip: Dielectrophoresis Applied to Microfluidic Platforms." Micromachines, 10(6), 423, 2019.

26. Y. S. Lu, Y. L. Liang, Huang, Y. P., Huang, J. Y., Lee, C., \& J. A. Yeh. "Assembly of Single Cells Array using Image Dielectrophoresis." Transducers 2007 - 2007 International Solid-State Sensors, Actuators and Microsystems Conference.

27. C. Qian, H. Huang, L. Chen, X. Li, Ge, Z., Chen, T., ... Sun, L. "Dielectrophoresis for Bioparticle Manipulation." International Journal of Molecular Sciences, 15(10), 18281-18309, 2014.

28. K. Khoshmanesh, S. Nahavandi, S. Baratchi, A. Mitchell, \& K. Kalantar-Zadeh. "Dielectrophoretic platforms for bio-microfluidic systems." Biosensors and Bioelectronics, 26(5), 1800-1814, 2011.

29. S. Gordon,\& P. R. Taylor. "Monocyte and macrophage heterogeneity." Nature Reviews Immunology, 5(12), 953-964, 2005.

30. Y. Pires-Afonso, S.P., Niclou, A. Michelucci. "Revealing and Harnessing Tumour-Associated Microglia/Macrophage Heterogeneity in Glioblastoma." Int J Mol Sci., 21;21(3), 2020.

31. F. J. Armistead, J. G. D. Pablo, H. Gadêlha, S. A. Peyman, \& S. D. Evans. "Cells Under Stress: An Inertial-Shear Microfluidic Determination of Cell Behavior.” Biophysical Journal, 116(6), 11271135,2019
32. Martinez-Duarte R, Renaud P, Madou MJ. A novel approach to dielectrophoresis using carbon electrodes. Electrophoresis. 2011 Sep;32(17):2385-92 .

33. Martinez-Duarte, R. (2014). SU-8 Photolithography as a Toolbox for Carbon MEMS. Micromachines, 5(3), 766-782.

34. Levis, M., Kumar, N., Apakian, E., Moreno, C., Hernandez, U., Olivares, A., ... Zartman, J. J. (2019). Microfluidics on the fly: Inexpensive rapid fabrication of thermally laminated microfluidic devices for live imaging and multimodal perturbations of multicellular systems. Biomicrofluidics, 13(2), 024111

35. Y. Yildizhan, N. Erdem, M. Islam, R. Martinez-Duarte, \& M. Elitas, "Dielectrophoretic Separation of Live and Dead Monocytes Using 3D Carbon-Electrodes", Sensors (Basel, Switzerland), 17(11), 2691, 2017

36. Islam, M., Natu, R., \& Martinez-Duarte, R. (2015). A study on the limits and advantages of using a desktop cutter plotter to fabricate microfluidic networks. Microfluidics and Nanofluidics, 19(4), 973985 . 